\title{
Memory priming and trial spacing effects in Pavlovian learning
}

\author{
CEYHUN SUNSAY, LEE STETSON, and MARK E. BOUTON \\ University of Vermont, Burlington, Vermont
}

\begin{abstract}
Conditioning trials that are massed in time produce less conditioning than those that are spaced in time. Four experiments with rat subjects examined whether a recent conditioning trial interferes with conditioning on the next trial by temporarily "priming" information in short-term memory (e.g., Wagner, 1978, 1981). We used appetitive conditioning procedures in which priming trials preceded target trials by $60 \mathrm{sec}$. When the priming trials were nonreinforced presentations of a conditioned stimulus (CS), the CS had to be the same CS as the one on the target trial to interfere with conditioning. When priming trials were actual CS-unconditioned stimulus (US) pairings, the CS identity did not matter; the US was the event that interfered with conditioning on the next trial. Reinforced trials reduced performance in a way that did not depend on context blocking. The results suggest that CS and US priming effects do contribute to conditioning deficits observed with massed trial procedures. The results are consistent with Wagner's (1981) "sometimes opponent process," or SOP, model, although a result that is paradoxical for the model suggests that recent USs may have motivational as well as memory effects.
\end{abstract}

Trial spacing has an important impact on learning. In classical conditioning, conditioning trials that are spaced in time produce better conditioning than trials that are massed in time (e.g., Barnet, Grahame, \& Miller, 1995; Pavlov, 1927; Rescorla \& Durlach, 1987; Terrace, Gibbon, Farrell, \& Baldock, 1975).

There are a number of mechanisms that can contribute to the trial spacing effect in classical conditioning (see Barela, 1999, for one recent review). Interest in trial spacing has been renewed in recent years because of claims that the animal's perception of time is central to conditioning. According to Gallistel and Gibbon (2000), the acquisition of conditioned responding depends on the animal deciding that the rate of reinforcement in the conditioned stimulus (CS) is higher than that in the background (cf. Gibbon \& Balsam, 1981). To implement the idea, the animal is assumed to calculate the ratio of the estimated rate of reinforcement in the CS over the rate of reinforcement in the absence of the CS. Algebraically, this is equivalent to calculating the ratio of time in the intertrial interval, $I$, over time in the CS, $T$. When the $I / T$ ratio exceeds a threshold, the animal will respond. Spacing conditioning trials in time increases the rate of conditioning because it decreases the rate of reinforcement in the background, as realized in a higher $I / T$ ratio.

Bouton and Sunsay (2003) examined predictions of this perspective in several partial reinforcement procedures. Such procedures intermix reinforced trials (trials in which the CS and an unconditioned stimulus [US] are paired) and nonreinforced trials (trials in which the CS is

This research was supported by Grant RO1 MH64847 from the National Institute of Mental Health to M.E.B. Correspondence should be addressed to M. E. Bouton, Department of Psychology, University of Vermont, Burlington, VT 05405 (e-mail: mark.bouton@uvm.edu). presented without the US). Most models of conditioning assume that the nonreinforced trials decrease associative strength (e.g., Rescorla \& Wagner, 1972) or add inhibition to the CS (Pearce \& Hall, 1980; Wagner, 1981). In contrast, the Gallistel-Gibbon and Gibbon-Balsam models assume that they have no such effect. Because $T$ is assumed to accumulate between successive reinforcers, adding nonreinforced trials to a continuous reinforcement procedure merely increases $T$ and thus decreases the $I / T$ ratio. However, Bouton and Sunsay (2003) observed two kinds of findings that challenge this approach. First, groups that had equal $I / T$ ratios conditioned at different rates depending on how $T$ was distributed over individual trials. That is, conditioning was affected by the number of nonreinforced trials, rather than merely accumulated $T$. Second, the temporal proximity of nonreinforced trials to the next reinforced trial made a difference: Nonreinforced CS presentations that occurred $60 \mathrm{sec}$ before the next reinforced trial hurt conditioning, whereas those that occurred more remotely in time (e.g., $240 \mathrm{sec}$ before the reinforced trial) did not. Neither finding is consistent with the Gallistel-Gibbon or Gibbon-Balsam models.

Bouton and Sunsay (2003) noted that their results were consistent with Wagner's (e.g., 1981) "sometimes opponent process" model, or "SOP," which accommodates both effects. The present experiments were designed to extend the second finding - that recent trials are especially damaging to conditioning on the next trial. SOP assumes that good learning on a particular trial requires that the CS and US both be maximally activated in a shortterm memory state at the same time. However, recent presentations of either the CS or the US can temporarily "prime" the representation of the event in short-term memory, making it less effective on its next presentation because it is less surprising (e.g., Wagner, 1978). For ex- 
ample, in the language of SOP, presentation of a nonreinforced trial would put the CS memory node into an active state (A1), but this would soon decay to a secondarily active state (A2). Because learning and performance on the next trial would depend on the CS being in A1 (and being processed with the US node also in the A1 state), a recent CS presentation that left the CS node in A2 would interfere with conditioning on the next trial. Theoretically, recent presentations of either the CS or the US would prime the corresponding memory node and make conditioning less effective.

It is possible that priming effects contribute to the detrimental effects of massed (as opposed to spaced) conditioning trials. However, priming has received surprisingly little attention in the recent literature. In an early report using rabbit eyeblink conditioning, Pfautz and Wagner (1976) found that nonreinforced CS presentations shortly before tests of a target CS suppressed responding to the target. Terry (1976) also demonstrated that proximal US presentations can hurt eyeblink conditioning. In flavor aversion learning, a recent exposure to the CS (Best \& Gemberling, 1977; Best, Gemberling, \& Johnson, 1979), US (e.g., Domjan, 1980), or CS-US pairing (Domjan, 1980) before a single conditioning trial reduces conditioning. However, little work on priming has been done in appetitive conditioning, where the models of Gallistel and Gibbon (2000) and Gibbon and Balsam (1981) have been most typically applied. The present experiments therefore examined the possible contribution of priming to trial massing deficits observed in the appetitive food cup entry preparation (Bouton \& Sunsay, 2003). This method is widely used in current studies of conditioning, and several recent investigators have used it to test predictions of the Gallistel-Gibbon and GibbonBalsam models (e.g., Bouton \& Sunsay, 2003; Haselgrove \& Pearce, 2003; Holland, 2000; Kirkpatrick \& Church, 2000; Lattal, 1999).

\section{EXPERIMENT 1}

The purpose of the first experiment was to study the priming effect observed by Bouton and Sunsay (2003), in which recent nonreinforced presentations of the CS reduced conditioning. As we already noted, the effect can be interpreted as a result of self-generated priming of the CS node into A2. However, the effect could also be due to some inhibitory aftereffect of the nonreinforced prime; if nonreinforcement generates frustration, for example, frustration might motivate an incompatible behavior like withdrawal from the food cup. Or the effect could be due to a blocking effect (Kamin, 1969) in which presentation of the CS on the priming trial signaled the US and made it less surprising when it was paired with the US on the target trial. In the language of SOP, the priming CS might work by putting the US node, rather than the CS node, into A2.

Experiment 1 was designed to separate these alternatives. The procedure is illustrated in Figure 1. Four groups received conditioning treatments in which CSs were paired with the US at the same points in time in a series of conditioning sessions. On half of these target trials, the CS was a tone, and on the other half, the CS was termination of the houselights. Two of the groups received nonreinforced priming trials that ended $60 \mathrm{sec}$ before each target trial. For Group Same 60, the prime was the same CS as the one in the upcoming target trial. For Group Diff 60, the prime was the different CS. In either group, the two CSs were reinforced on half their presentations and served as nonreinforced primes on the other half. Both groups also received reinforced trials in the potential presence of an inhibitory aftereffect, or retrievalgenerated priming of the US into A2. But only Group Same 60 received a reinforced trial $60 \mathrm{sec}$ after the target CS had been primed.

Analogous groups received same and different primes $240 \mathrm{sec}$ before each target trial. On the basis of earlier results (Bouton \& Sunsay, 2003, Experiment 3), we expected that $240 \mathrm{sec}$ would be sufficient to allow any effect of the prime to decay by the time of the targetreinforced trial. If conditioning in this preparation is affected by self-generated priming of the CS, then Group Same 60 should show less conditioned responding than any other group.

\section{Method}

Subjects. The subjects were 32 female Wistar rats obtained from Charles River, Inc., St. Constance, Quebec. They were approximately 85-90 days of age at the start of the experiment and were individually housed in suspended stainless steel cages in a room maintained on a 12:12-h light:dark cycle. The rats were food-deprived to $80 \%$ of their free-feeding weights throughout the experiment.

Apparatus. Two sets of four Skinner boxes were housed in sound attenuation chambers located in separate rooms of the laboratory. Although the two sets were designed to function as different contexts, they were not used in that capacity here. The boxes in both sets measured $31.75 \times 24.13 \times 29.21 \mathrm{~cm}(1 \times \mathrm{w} \times \mathrm{h})$. The sidewalls and ceiling were made of clear acrylic plastic, while the front and rear walls were made of brushed aluminum. Recessed $5.08 \times 5.08 \mathrm{~cm}$ food cups were centered in the front wall at about the level of the floor. These cups had infrared photo beams positioned approximately $1.2 \mathrm{~cm}$ behind the plane of the wall and $1.2 \mathrm{~cm}$ above the bottom of the cup. In one set of four boxes, the floor was composed of stainless steel grids $(0.48 \mathrm{~cm}$ in diameter) spaced $3.18 \mathrm{~cm}$ center-tocenter. One sidewall had black horizontal stripes, $3.81 \mathrm{~cm}$ wide and $3.81 \mathrm{~cm}$ apart. The ceiling had similarly-spaced stripes oriented in the same direction. A distinctive odor was created by leaving a cup of about $10 \mathrm{ml}$ of a $2 \%$ McCormick anise extract solution in a dish outside the front wall of the Skinner box. In the other set of boxes, the floor was made of alternating stainless steel grids of different diameters $(0.48$ and $1.27 \mathrm{~cm})$ separated by $1.59 \mathrm{~cm}$ center-to-center. One sidewall and the ceiling were covered with rows of dark dots (1.9- $\mathrm{cm}$ diameter) with the adjacent dots separated by about $1.27 \mathrm{~cm}$. A distinctive odor was created by leaving a cup of about $10 \mathrm{ml}$ of a $4 \%$ McCormick coconut extract solution in a dish outside the front wall of the Skinner box. Two CSs were used. One CS was a $10-\mathrm{sec} 3000-\mathrm{Hz}(80 \mathrm{dBA})$ tone delivered through a $7.6-\mathrm{cm}$ speaker mounted to the ceiling of the sound attenuation chamber (background noise was maintained at $60 \mathrm{dBA}$ ). The other CS consisted of terminating the houselights (two 7.5-W incandescent bulbs that were mounted to the ceiling of the sound attenuation chamber). The US was two 45-mg food pellets (Improved Formula A, Noyes, Lancaster, $\mathrm{NH}$ ) delivered at the termination of the $\mathrm{CS}$. 


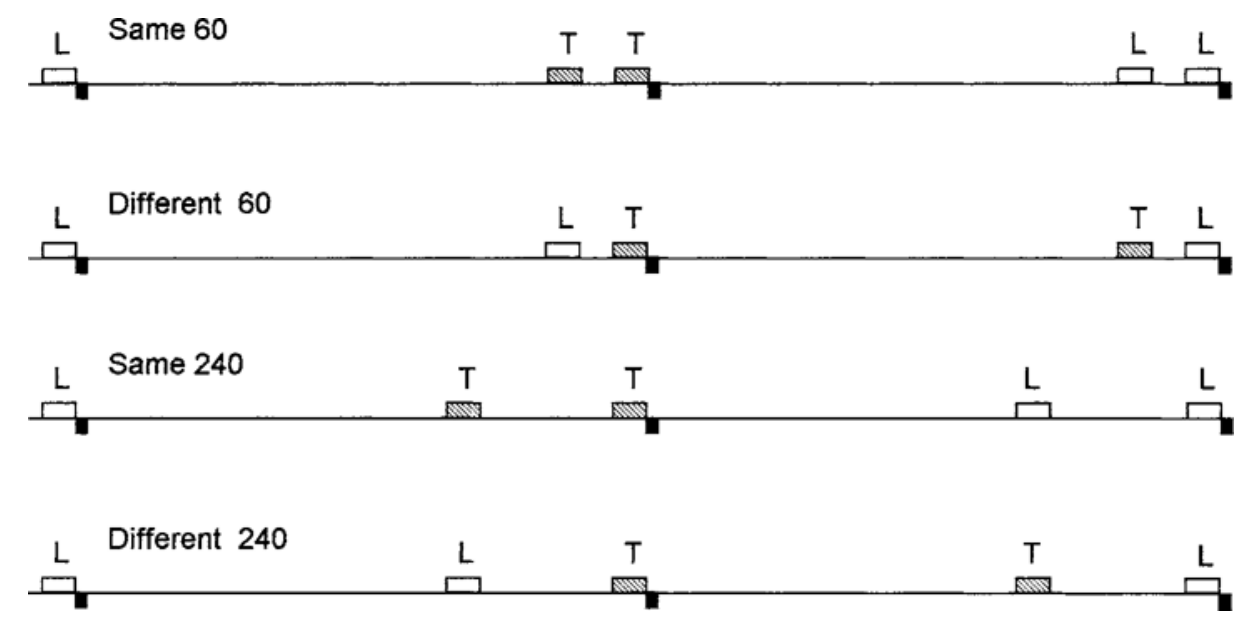

Figure 1. Design and procedure of Experiment 1. Time (not to scale) is indicated left to right; different groups are indicated by different lines. Boxes above the line indicate CSs (tone or light); black boxes represent US (food) presentations.

Procedure. The experiment began with an initial 20-min magazine training session in which the rats learned to retrieve pellets from the food cups. Each rat received $30-40$ pellets in its assigned box in this initial session.

The rats were then randomly assigned to four groups $(n=8)$. As illustrated in Figure 1, all groups received a mixture of both reinforced target trials and nonreinforced priming trials. There were four reinforced trials and four nonreinforced trials in each session. All CS presentations were $10 \mathrm{sec}$ in duration. For all groups, the interval between USs was $1,080 \mathrm{sec}$, and the CS that preceded each US alternated between tone and light-off (TLTL on some sessions and LTLT on others in an ABBA order). Two groups (Same 60 and Diff 60) received a 60-sec gap between the end of each prime and the onset of the target, whereas the other two groups (Same 240 and Diff 240) received a 240-sec gap. For Groups Same 60 and Same 240, the prime CS and succeeding target were always the same CS, whereas for Groups Diff 60 and Diff 240, the prime CS and target were always different. Notice that each group received a $50 \%$ reinforcement schedule with both the tone and the light; what made the groups different was the trial sequence and spacing. There were a total of 18 daily 72 -min sessions.
On the target trials, the computer recorded the number of photobeam breaks during each 10 -sec CS and during the 10 -sec interval that immediately preceded it (the pre-CS period). Each trial's data were then converted into "elevation scores" by subtracting the number of responses made during the pre-CS period from the number of responses made during the CS. Elevation scores and pre-CS responses were always analyzed with identical analyses of variance (ANOVAs), with a rejection criterion of $p<.05$.

\section{Results}

Responding on the target trials is summarized in Figure 2. The figure suggests that conditioning was slowest in Group Same 60. A mixed prime-type (same or different $) \times$ gap duration $(60$ vs. $24 \mathrm{sec}) \times$ stimulus $(\mathrm{T}$ vs. $\mathrm{L})$ $\times$ trial-block ANOVA revealed no interactions with the stimulus factor $[F \mathrm{~s}(1,28)<1]$, and so the figure collapses over stimulus. There was, however, a main effect of stimulus $[F(1,28)=50.65]$, confirming that the tone evoked more responding than the light-off. There were main ef-

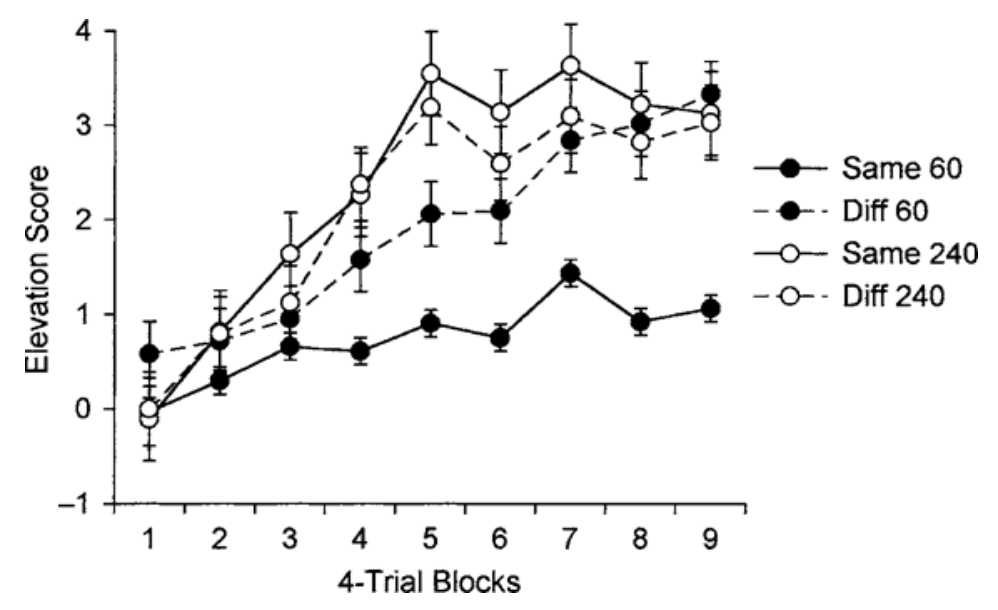

Figure 2. Mean elevation scores $( \pm 1 S E M)$ of the groups during conditioning in Experiment 1. 
fects of trial block $[F(8,224)=23.12]$ and gap duration $[F(1,28)=6.13]$, which also interacted $[F(8,224)=$ 2.76]. The main effect of prime type was not reliable $[F(1,28)=1.56]$, but the prime type $\times$ gap duration interaction approached significance $[F(1,28)=2.68$, $p=.06]$. A planned comparison between Groups Same 60 and Diff 60 (collapsing over trial blocks) revealed a significant difference between them $[F(1,28)=5.07]$. The corresponding difference between Groups Same 240 and Diff 240 was not significant $[F(1,28)<1]$. Groups Same 60 and Same 240 also differed $[F(1,28)=11.21]$. No other interaction approached significance [largest $F(8,224)=1.62]$. The lack of any interaction with the stimulus factor indicates that the strength of the difference between the same and diff primes did not depend on whether the tone or light-off was the target or the prime.

To explore the interaction with trial block, we performed an identical ANOVA that isolated the last five blocks of acquisition. This ANOVA revealed a main effect of gap duration $[F(1,28)=6.88]$, but not prime type $[F(1,28)=1.64]$. The gap duration $\times$ prime type interaction, however, was significant $[F(1,28)=4.24]$, as was a pairwise comparison between Groups Same 60 and Diff $60[F(1,28)=5.59]$. The difference between Groups Same 240 and Diff 240 was not significant $[F(1,28)<1]$. The results leave little doubt that at the shorter 60-sec gap duration, the priming with the same CS interfered more with conditioning than did priming with a different CS. Group Same 60 also responded significantly less than Group Same $240[F(1,28)=11.53]$.

The overall mean pre-CS scores on the target trials for Groups Same 60, Diff 60, Same 240, and Diff 240 were $2.19,2.06,1.51$, and 1.01 , respectively. The ANOVA revealed neither a prime type effect $[F(1,28)=1.64]$ nor a prime type $\times$ gap duration interaction $[F(1,28)<1]$. Thus, the crucial difference in CS responding between Groups Same 60 and Diff 60 was not compromised by confounded differences in pre-CS scores. The pre-CS analysis did reveal an effect of block $[F(8,224)=8.47]$ and gap duration $[F(1,28)=8.20]$. The block $\times$ gap duration interaction was also reliable $[F(2,224)=2.32]$, which is consistent with a growing effect of gap. Thus, particularly late in training, responding during the preCS period following a recent prime presentation (60-sec conditions) was higher than that following a more remote prime (240-sec conditions). Analysis of the last five blocks supported the same conclusions: block effect $[F(4,112)=5.97]$, gap duration effect $[F(1,28)=$ 11.41], and prime type and prime type $\times$ gap duration effects $[F \mathrm{~s}(1,28)<1]$. No other effects in either analysis approached reliability $(p s \geq .20)$.

\section{Discussion}

The results replicate the priming effect reported by Bouton and Sunsay (2003). However, they also indicate that to obtain the maximal effect, the priming CS must be the same as the target CS. When they were different (Group Diff 60), there was no evidence of significant priming, suggesting little (if any) role for blocking or an inhibitory aftereffect of the prime. The similarity of conditioning in Groups Same 240 and Diff 240 is consistent with other data suggesting that the effect of the nonreinforced prime is over by $240 \mathrm{sec}$ (Bouton \& Sunsay, 2003, Experiment 3). The results are thus consistent with the idea that a nonreinforced presentation of the CS $60 \mathrm{sec}$ before target trials produces a short-term effect that specifically reduces conditioning with the CS itself. They are uniquely consistent with the models of Wagner (1978, 1981).

It is worth observing that the results further challenge time-accumulation models of conditioning (Gallistel \& Gibbon, 2000; Gibbon \& Balsam, 1981). Those models assume that the rate of conditioning is proportional to the ratio between $I$ and $T$, which is assumed to accumulate. In the present design, Groups Same 60 and Same 240 had equal $I / T$ ratios. The fact that they differed significantly during conditioning is thus not consistent with the Gallistel-Gibbon and Gibbon-Balsam models (see also Bouton \& Sunsay, 2003). Interestingly, the groups also differed in the number of four-reinforcer blocks required to reach the acquisition criterion measure used by Bouton and Sunsay (three consecutive blocks with elevation scores calculated with a 30 -sec base $>3.0)[U(8,8)=$ 7.5]. Groups Same 60 and Diff 60 required a median of 8.0 and 5.0 blocks to reach criterion, respectively. This result may be especially interesting, because it is a direct test of time-accumulation models' prediction that $I / T$ influences the number of reinforcers it takes the organism to "decide" to respond.

\section{EXPERIMENT 2}

Although the results of Experiment 1 encourage an SOP account of trial spacing, previous studies of trial spacing effects have generally employed continuous reinforcement procedures - that is, procedures in which every CS presentation is paired with a US - rather than partial reinforcement schedules. In the interest of a general understanding of the role of priming in trial spacing effects, we therefore turned to the possible priming effects of reinforced (as opposed to nonreinforced) trials. Although the CS priming mechanism identified in Experiment 1 might certainly play a role in continuous reinforcement procedures, the presentation of the US itself on a priming trial would also have important consequences. In SOP, the US would temporarily prime itself into A1 and then A2. Experiment 2 therefore asked whether the identity of the priming CS still matters when the priming trials are reinforced.

\section{Method}

Subjects and Apparatus. The subjects were 16 female Wistar rats obtained from the same breeder as before. They were approximately 85-90 days of age at the start of the experiment, and their housing and maintenance were the same as in the preceding experiment. The apparatus was also the same. One CS was the same tone. The other CS also involved termination of the houselights. How- 


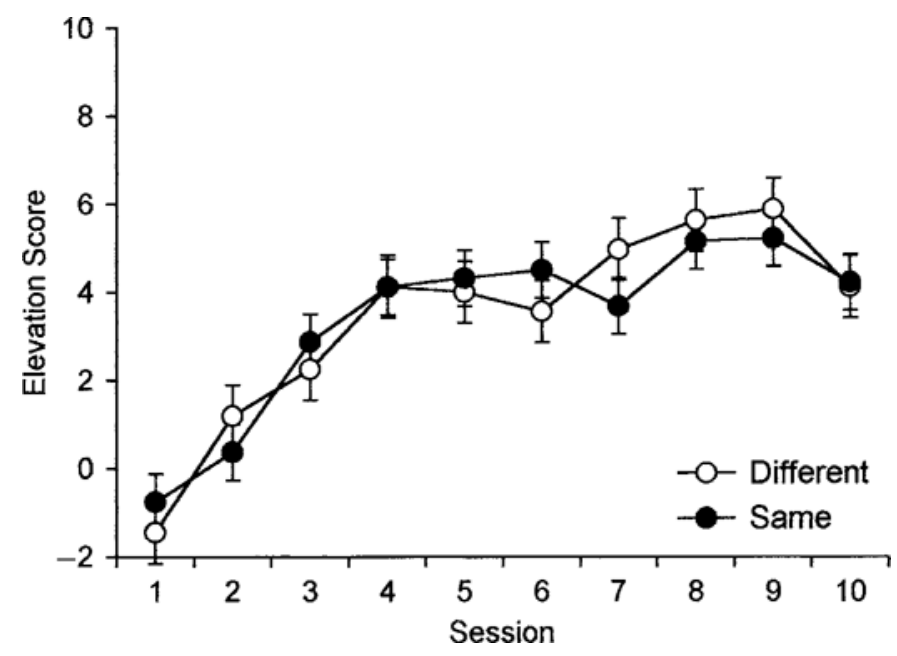

Figure 3. Mean elevation scores $( \pm 1 S E M)$ of the groups during conditioning in Experiment 2.

ever, in an effort to make it more salient, the houselights were flashed ( $0.5 \mathrm{sec}$ on; $0.5 \mathrm{sec}$ off $)$. The US was the same.

Procedure. The experiment began with an initial 20-min magazine training session in which the rats learned to retrieve pellets from the food cups. Each rat received 32-33 pellets in this session. They were then randomly assigned to two groups $(n=8)$. The groups received the trial schedule used by Groups Same 60 and Diff 60 in Experiment 1, with the only difference being that the prime trials all ended in a US. There were 10 sessions run in 5 days (two sessions a day, separated by approximately $80 \mathrm{~min}$ ). Session duration was $72 \mathrm{~min}$.

\section{Results}

Responding on the target trials (collapsing over the tone and light stimuli) is summarized in Figure 3. A group $\times$ stimulus $\times$ session ANOVA of the trial blocks revealed a significant session effect $[F(9,126)=20.49]$. The stimulus effect was also marginally significant $[F(1,14)=4.33$, $p=.056]$. Once again, conditioning with tone resulted in higher elevation scores. The critical group effect and the group $\times$ session interaction did not approach significance $(F \mathrm{~s}<1, p \mathrm{~s}>.78)$. No other effects were reliable [largest $F(9,126)=1.37]$, including interactions with the stimulus factor, which justified collapsing over that variable in Figure 3.

An identical analysis with the pre-CS scores revealed a significant effect of session $[F(9,126)=3.19]$. Responding in the pre-CS period decreased and then increased over sessions. The stimulus effect $[F(1,14)=4.67]$ and the stimulus $\times$ group interaction $[F(1,14)=8.10]$ were also significant. There was more pre-CS responding on trials with the tone CS in Group Same, but not in Group Diff; the pattern presumably reflects Type I error. No other effects were reliable $[F(1,14)=1.93]$. The mean pre-CS scores for the tone CS were 1.28 and 2.23 for Groups Diff and Same, respectively; for the light CS, they were 1.36 and 1.63 .

Because we were reluctant to accept the null hypothesis, we also analyzed the results of two other dependent measures that were available - the total time spent in the food cup and the latency to the first food cup entry during the CS. (Temporal measures of food-cup entry have sometimes been used in other laboratories, e.g., Fox \& Holland, 1998; Kaye \& Mackintosh, 1990.) Analyses of these measures also failed to uncover group effects or group $\times$ session interactions [time in cup, $F_{\mathrm{s}}(1,14)<$ 1.58; latency to first magazine entry, $F_{\mathrm{s}}(1,14)<2.72$ ]. Averaging over all trial blocks, the mean time in the cup during the CS was 2.86 and $3.25 \mathrm{sec}$ for Groups Same and Diff, respectively, and latency to first entry was 2.92 and $3.48 \mathrm{sec}$.

\section{Discussion}

The results provide no support for the idea that the identity of the CS matters when the priming trial is reinforced. This result, which was evident in three dependent measures, suggests that the CS priming mechanism identified for nonreinforced primes in Experiment 1 may not be as important when a US is added to the priming trial. One possibility is that if short-term memory has a limited capacity, so that a limited number of events can be held in the memory buffer (A2) for any length of time (e.g., Wagner, 1981; see also Wagner, 1978), then presentation of the US after the CS might displace the primed CS representation from short-term memoryand thus render CS priming less effective. That is, the US might serve as a distractor that reduces CS priming (cf. Best et al., 1979; Terry, 1976; Whitlow, 1975). In that scenario, although the US would cancel the effect of the $\mathrm{CS}$, it could itself remain primed and available to influence the next trial. We tested for such an effect in the next experiment.

\section{EXPERIMENT 3}

The third experiment was designed to compare the effectiveness of three different types of priming trials. Dif- 


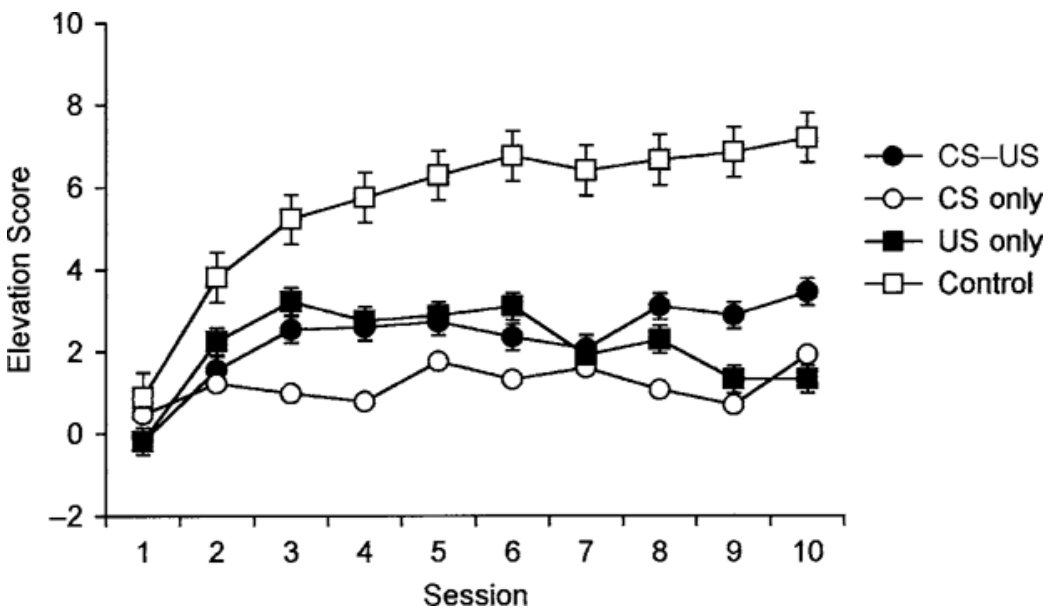

Figure 4. Mean elevation scores ( $\pm 1 S E M$ ) of the groups during conditioning in Experiment 3.

ferent groups received CS-alone, US alone, and reinforced (CS-US) primes. All groups received target CSUS pairings spaced 1,290 sec apart. Between these trials, the groups also received an additional CS-US pairing, a CS-alone presentation, and a presentation of the US alone. Group Control received the events at least $550 \mathrm{sec}$ before the target CS-US pairing - an interval presumably long enough to preclude priming at the time of the target trial. The remaining groups each had different events ending $60 \mathrm{sec}$ before the target trial. In Group CSonly, the event that occurred in the priming position was a nonreinforced presentation of the CS; in Group USonly, the event in the priming position was a US presentation; and in Group CS-US, the event was the CS-US trial. Each group received its remaining events more remotely in time, at least $540 \mathrm{sec}$ before the target trial. The groups thus received equivalent exposures to target trials and intertarget events, but differed especially in which event occurred $60 \mathrm{sec}$ before the target trial.

\section{Method}

Subjects and Apparatus. The subjects were 32 female Wistar rats of the same age and stock as in the preceding experiments. The apparatus was also the same. The CS was the tone stimulus that was used in Experiment 1. The US was the same as well.

Procedure. The experiment began with an initial 20-min magazine training session in which the rats received 33-36 pellets in the apparatus. They were then randomly assigned to four groups ( $n=$ 8 ). All groups received 10 daily 86 -min sessions that contained four target tone-US pairings separated by $1,290 \mathrm{sec}$. The groups also received a US, a presentation of the tone alone, and another tone-US pairing between each target trial. Group CS-only received the tone presentation (a CS-only trial) ending $60 \mathrm{sec}$ before each target trial; US alone and CS-US pairings occurred more remotely in time (800 and $540 \mathrm{sec}$ before the target trial). Group CS-US received a tone-US pairing $60 \mathrm{sec}$ before each target trial, and CS-only and US-only presentations more remotely ( 800 and $540 \mathrm{sec}$ before the target). Group US-only received a US (two food pellets) $60 \mathrm{sec}$ before each target trial and CS-US and CS-only trials 790 and $540 \mathrm{sec}$ before the target. Finally, Group Control had its extra events (US only, CS-US pairing, and CS only) $1,050,800$, and $550 \mathrm{sec}$ before the target trials. As usual, data analyses focused on responding during the target trials and the immediately preceding pre-CS period.

\section{Results}

The results are presented in Figure 4, which shows the mean elevation scores of each group during the target trials of each session (four-trial block). A group $\times$ session ANOVA revealed reliable effects of group $[F(3,28)=$ $11.39]$, session $[F(9,252)=8.28]$, and a group $\times$ session interaction $[F(27,252)=3.12]$. Planned comparisons examined the group effect. While Group Control differed from each of the primed groups [smallest $F(1,28)=$ 20.89 ], the primed groups did not differ [largest $F(1,28)=$ 2.44]. Evidently, priming with the CS alone, the US alone, or a CS-US pairing had comparably detrimental effects on responding on the target trials.

An identical analysis of responding during the pre-CS periods of the target trials suggested no differences among the groups. There was a session effect $[F(9,252)=2.65]$, with responding increasing over sessions. However, neither the group effect $[F(3,28)=1.39]$ nor the group $\times$ session interaction $[F(27,252)=1.48]$ was significant. The mean pre-CS scores averaged over the phase were 1.62, 2.00, 2.66, and 1.69 in Groups Control, CS-only, US-only, and CS-US, respectively.

\section{Discussion}

The results again replicated our previous findings with CS-only priming trials (Experiment 1 and Bouton \& Sunsay, 2003). However, they further indicate that US alone and CS-US pairings are also effective at reducing conditioning in this preparation. The effects of CS and US primes are straightforward. In the language of SOP, the CS-alone prime would put the CS node in A2 and the US prime would have done the same with the US node. In the case of the US prime, it is also possible that the US caused some conditioning of the context that caused partial blocking when the CS was reinforced on the next tar- 
get trial. (In SOP, blocking by context would be conceptualized as a case of retrieval-generated priming of the US.) Since all groups received the same USs between the crucial target trials, a context-blocking explanation would necessarily emphasize differential extinction of the context over 1,050 sec in Group Control and $60 \mathrm{sec}$ in Group US-only. There was no corresponding difference in the pre-CS rates, a potential measure of context conditioning. Nonetheless, we cannot rule out a contextblocking interpretation, and therefore consider it further in Experiment 4.

It is interesting that the CS-US prime did not produce more attenuation of conditioning than presenting either the CS or the US alone. The presentation of two events that each damaged conditioning on their own might be expected to produce even more damage when presented together. One possibility is that the primed groups were near a response floor that made detection of group differences difficult. It is worth noting that the elevation scores of the primed groups differed significantly from a hypothetical population mean of zero $[t(23)=7.03]$; there was thus potentially room on the response scale to detect group differences. Another, perhaps more interesting, possibility is the one suggested in Experiment 2: Presentation of the US in Group CS-US could have served as a distractor that nullified the effect of the CS prime, leaving the US the sole source of priming after the CS-US prime. The fact that the identity of the prime made no difference in Experiment 2 further suggests that CS priming plays little role in the effect of CS-US primes. The results of Experiments 2 and 3 are thus consistent in suggesting that the US presentation on the preceding trial is the factor that causes weak conditioning in massed-trial continuous reinforcement procedures.

\section{EXPERIMENT 4}

Experiment 4 had two purposes. First, it asked whether reinforced primes can reduce conditioned responding in a way that can be separated from context blocking. That is, is the priming effect of a recent US merely a result of context conditioning it creates, causing blocking at the time of reinforcement on the next trial? Second, the experiment asked whether primes can have an effect on performance, rather than learning, on the target trial.

Earlier research suggests that primes may influence both learning and performance. In one of their experiments, Bouton and Sunsay (2003) tested rats with massed and spaced trials after training with a massed or spaced conditioning procedure. They found both an enduring effect of trial massing (two partially reinforced groups trained with massed or spaced trials continued to differ regardless of spacing during testing) and a temporary effect in which trials that were massed during testing evoked less responding than trials that were spaced. The results thus suggested effects on both learning and performance. In their early priming experiment on rabbit eyelid conditioning, Pfautz and Wagner (1976) dem- onstrated an effect of nonreinforced trials on performance. They first ran conditioning to asymptote and then tested CSs after same or different nonreinforced primes. There was less responding after a same than a different trial. Unfortunately, our own unpublished efforts using a method analogous to that of Pfautz and Wagner were unsuccessful. After training to asymptote in the present conditioning preparation, primes corresponding to the ones studied in Experiments 1 and 3 had no consistent attenuating effect on performance.

It is possible that performance at asymptote, as tested in the earlier experiments, is somewhat less vulnerable to disruption by priming than performance at an earlier point in training. That is, our failure to find priming effects on responding after asymptotic training does not rule out performance effects earlier during conditioning. Therefore, Experiment 4 was designed to test a possible performance effect of recent reinforced trials with a novel within-subjects procedure. A single group of rats received continuous reinforcement with a tone CS in a trial schedule that intermixed intertrial intervals (ITIs) of 60, 240, and $960 \mathrm{sec}$. If CS-US primes affect performance rather than learning, we should expect less performance on those trials that followed the preceding trial by $60 \mathrm{sec}$ than on those that followed longer ITIs. However, because all subjects had conditioning with only a single CS, any effect of trial spacing on learning about the CS would be reflected in performance at all ITIs. Blocking is ordinarily assumed to influence learning rather than performance (e.g., Rescorla \& Wagner, 1972; Wagner, 1981). Thus, attenuated performance during the 60 -sec ITI would implicate a performance effect that would not be explicable in terms of context blocking.

\section{Method}

Subjects. There were 24 female Wistar rats $85-100$ days old and from the same supplier as in previous experiments. They were housed and maintained as before. The apparatus was also the same. In this experiment, the CS was a 30 -sec presentation of the tone. As usual, the US was two 45-mg food pellets.

Procedure. The experiment began with an initial 20-min magazine training session in which each rat received $30-35$ pellets. There were then nine daily 67.5-min acquisition sessions. Each session contained nine trials separated by a long, medium, or short ITI. The mean duration of these ITIs was 960,240 , and $60 \mathrm{sec}$, respectively; each varied by $\pm 25 \%$. The distribution of ITIs was pseudorandom with the constraint that there were always three long, medium, and short ITIs in each session and no like intervals could occur consecutively. In addition, over each block of three sessions, the number of different ITI transitions (long-short, medium-short, long-medium, short-medium, medium-long, and short-long) was equal (there were four of each).

\section{Results}

The results are presented in Figure 5. An ITI $\times$ session ANOVA on elevation scores showed significant main effects of session $[F(8,184)=20.17]$ and ITI $[F(2,46)=15.81]$. The interaction was not significant $[F(16,368)<1]$. Planned comparisons showed that both long and medium ITIs had significantly higher elevation scores than did the short ITI [smallest $F(1,46)=6.44$ ] 


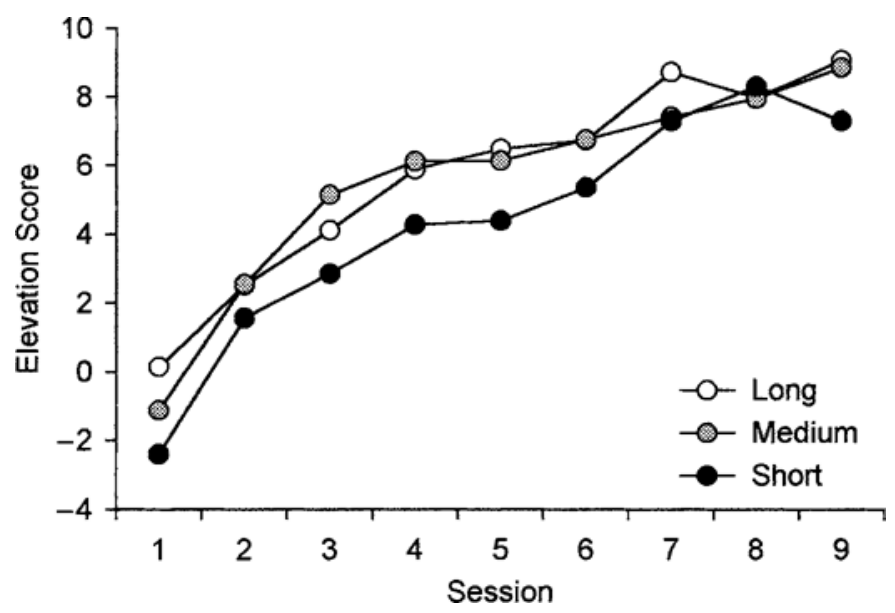

Figure 5. Mean elevation scores during trials in 960-sec (long), 240-sec (medium), and 60-sec (short) ITIs during conditioning in Experiment 4.

The long and medium ITIs had comparable elevation scores $[F(1,46)<1]$.

An identical analysis of pre-CS scores showed a significant main effect of session $[F(8,184)=2.29]$, but no ITI main effect $[F(2,46)=2.27]$. The session $\times$ ITI interaction was also significant $[F(16,368)=2.07]$. An ITI effect was evident only on the first session; the interaction did not approach significance when the first session was excluded $[F(14,322)<1]$. The mean pre-CS scores were 4.03, 3.67, and 3.53 for the short, medium, and long ITIs, respectively.

Figure 6 breaks the results down a different way. It shows responding on trials when the current ITI was short, medium, or long as a function of the duration of the preceding ITI. This sort of analysis asks the further question of whether responding on a given trial is also sensitive to the associative impact of the preceding ITI. For example, if a long ITI allowed an especially large boost in associative strength of the CS, then the boost might be detected as higher responding when the CS is presented on the next trial. However, the figure suggests no evidence that responding to the CS on trial $n$ was affected by the ITI on trial $n-1$. For purposes of analysis, the ITI on trial $n-1$ was categorized as either "long" or "short." The data were analyzed with a 2 (previous ITI: "long" or "short") $\times 3$ (current ITI) $\times 3$ (session block) ANOVA. This ANOVA showed a significant effect of current cycle only $[F(2,46)=15.07]$. Previous ITI had no effect on responding $[F(1,23)=2.38]$. The interaction between these factors was not significant $[F(2,46)<1]$. The

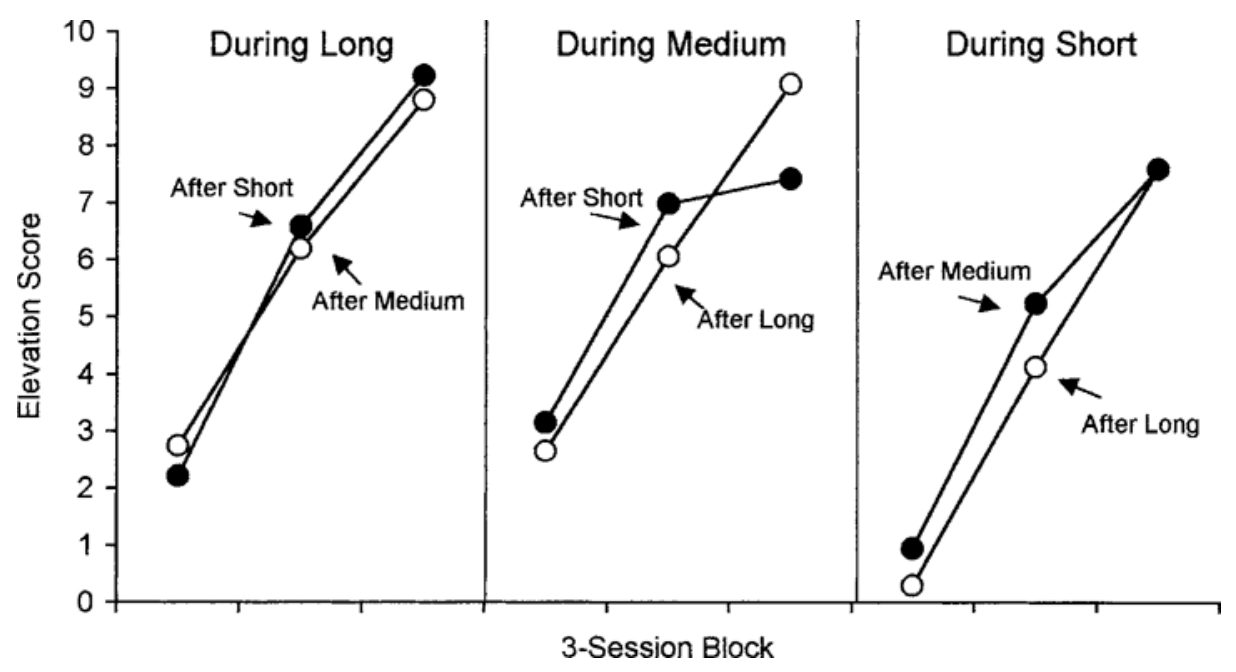

Figure 6. Mean elevation scores during conditioning in Experiment 4 as a function of ITI on both the current trial ("during") and the preceding trial ("after"). 
session effect was reliable $[F(2,46)=26.66]$, but did not interact with current ITI $[F(4,92)=1.0]$, previous ITI $[F(2,46)=2.49]$, or their interaction $[F(4,92)=1.96]$.

\section{Discussion}

These results indicate that short ITIs in a continuous reinforcement schedule can suppress performance. Such an effect appeared to be evident after the 60 -sec ITI only; it is interesting that there was no difference between the 240- and 960-sec ITIs. The latter finding continues to be consistent with the idea that the short-term priming effects we have observed in this preparation are gone within $240 \mathrm{sec}$. Notice that the effect of current ITI cannot be explained by the possibility that recent reinforced trials generate context conditioning that can block conditioning on the next trial. Any effect on learning should affect performance on all trials, regardless of the current ITI.

Although SOP can presumably allow priming effects on either learning or performance (a performance effect is suggested by Pfautz \& Wagner, 1976), it is interesting to note that the results pose something of a paradox for the model. During a short ITI, if the US node is truly in the A2 state, then it should be reflected in behavior. This follows from the idea that the CS elicits a conditioned response because it activates the US node into the A2 state. The paradox can be stated as follows: If the priming effect evident here is the result of the US being primed into A2, why should this suppress, rather than enhance, performance elicited by the CS through activation of the US node to A2? One possibility is that the conditioned response is not a linear function of the extent to which the US is in the A2 state; the mechanics of response production might not be the same as those that allow the US to produce priming. Priming might also reflect other kinds of processes. For example, it is possible that a signal for food might have more motivational impact on behavior in the hungry rat if food has not been presented recently.

\section{GENERAL DISCUSSION}

The results of the present experiments are consistent with the idea that recent trials can contribute to a trial massing deficit by causing short-term "priming" effects on conditioned responding. In Experiments 1 and 3, a nonreinforced presentation of the CS that ended $60 \mathrm{sec}$ before a reinforced trial produced a decrease in conditioning. This effect was not created by a different CS and was thus not a result of some inhibitory aftereffect of a nonreinforced trial or blocking. Furthermore, because the priming effect was stronger when the gap between it and the target was 60 rather than $240 \mathrm{sec}$, it was not consistent with the idea that the rate of conditioning is merely a function of the ratio of the ITI and the accumulated time in the CS during interreinforcer cycle-for example, the $I / T$ ratio (Gallistel \& Gibbon, 2000; Gibbon
\& Balsam, 1981). The simplest explanation appears to be the one captured by SOP: A recent presentation of the CS makes the CS less surprising on the next trial, reducing the performance and conditioning possible on the next trial.

Other results suggest that the CS priming effect is most likely to play a role in massed-trial procedures that intermix reinforced and nonreinforced trials. In continuous reinforcement procedures, where recent priming trials are reinforced rather than nonreinforced, the presence of the US appears to reduce the possible contribution of the CS priming mechanism (Experiments 2 and 3). Although a reinforced priming trial $60 \mathrm{sec}$ before the target clearly produced a deficit in conditioning (Experiments 3 and 4), there was little impact of adding a CS to a US prime alone (Experiment 3), and the identity of the CS did not matter (Experiment 2). Thus, the recent reinforced trials created a deficit in conditioning that results mainly from presence of the US, which is alone sufficient to interfere with conditioning (Experiment 3 ).

There are several ways to understand the impact of the US prime. First, the US presentation would put the US node into A1, which would then decay to A2, and for some period of time thereafter produce a self-generated priming effect. A role for self-generated US priming has been identified in eyeblink conditioning by Terry (1976). Domjan (1980) also suggested a similar role for proximal US exposure in taste aversion learning. Second, it is conceivable that a recent US might somehow inflate the animal's estimate of the background rate of reinforcement, which might then decrease conditioned responding in the style anticipated by Gallistel and Gibbon (2000). However, the Gallistel-Gibbon model provides no way for a recent US to have a particular effect on the animal's perception of its rate. Instead, the animal is assumed to calculate the rate of reinforcement as a running average. In the present experiments, animals showing US priming effects had the same number of reinforcers in sessions of the same duration, and thus the same average reinforcement rate, as did controls. The GallistelGibbon model is no better at predicting the proximal US effect evident in Experiments 3 and 4 than it was at explaining the proximal CS effect evident in Experiments 1 and 3 (see also Bouton \& Sunsay, 2003).

A third explanation of the impact of the priming US is context blocking. Any US presentation would theoretically increase the associative strength of the context, and this could at least partially block conditioning of the CS when the CS is next reinforced. In the language of SOP, the context would put the US node in A2 via retrievalgenerated priming. Longer intervals between the priming US and the target trial would presumably allow extinction of the context. Context-blocking mechanisms are known to influence conditioning, as suggested by research on the US preexposure effect (e.g., Randich \& Ross, 1985), and it seems likely that they would contribute to trial massing effects in many experiments. 
However, Experiment 4 uncovered two types of evidence suggesting an additional mechanism. First, there was a suppressive effect of a recent reinforced trial on performance. Context blocking has an effect on learning, which would have a general impact on responding to the CS at all ITIs. Second, there was no detectable difference in impact of long and short ITIs when responding was assessed on the next trial (Figure 6). That result suggests that, with the methods used here, the detrimental impact of a reinforced trial experienced $60 \mathrm{sec}$ ago is quite specific to the current interval.

We noted that the results of Experiment 4 are instead most consistent with an effect of recent USs on performance. Interestingly, little of the data we have presented here necessarily indicates that priming also affects learning; Pfautz and Wagner's (1976) early results likewise emphasized an effect on performance. Nonetheless, our earlier experiments (Bouton \& Sunsay, 2003) suggested that primes may affect both performance and learning. Either result is consistent with SOP principles, except for the paradox we noted before: It is hard to see why a US node in A2 would interfere with performance that would otherwise result from the CS putting the US node into that very state. We have noted that the mechanics of response generation might intervene to make priming and response generation differentially affected by the extent to which the US is in A2. An alternative possibility recognizes that the present experiments involved delivery of food USs to hungry rats. Increased time since the last food pellet might make the animal especially excited when the CS comes on again. Alternatively, the animal might learn that the food pellet has especially high incentive value when the trial has been delayed for a while (see Dickinson \& Balleine, 1994). It seems possible that motivational processes, in addition to memory processes (Wagner, 1978, 1981), might contribute to the priming effects that the present results suggest can play a role in causing trial massing effects in appetitive conditioning.

\section{REFERENCES}

Barela, P. B. (1999). Theoretical mechanisms underlying the trial spacing effect in Pavlovian conditioning. Journal of Experimental Psychology: Animal Behavior Processes, 25, 177-193.

BarNet, R. C., Grahame, N. J., \& Miller, R. R. (1995). Trial spacing effects in Pavlovian conditioning: A role for local context. Animal Learning \& Behavior, 23, 340-348.

Best, M. R., \& Gemberling, G. A. (1977). The role of short-term processes in the CS preexposure effect and the delay of reinforcement gradient in long-delay taste-aversion learning. Journal of Experimental Psychology: Animal Behavior Processes, 3, 253-263.

Best, M. R., Gemberling, G. A., \& Johnson, P. E. (1979). Disrupting the conditioned stimulus preexposure effect in flavor-aversion learning: Effects of interoceptive distractor manipulations. Journal of Experimental Psychology: Animal Behavior Processes, 5, 321-334.

Bouton, M. E., \& Sunsay, C. (2003). Importance of trials versus accumulating time across trials in partially reinforced appetitive conditioning. Journal of Experimental Psychology: Animal Behavior Processes, 29, $62-77$.
Dickinson, A., \& Balleine, B. W. (1994). Motivational control of goal-directed action. Animal Learning \& Behavior, 22, 1-18.

Domuan, M. (1980). Effects of intertrial interval on taste aversion learning in rats. Physiology \& Behavior, 25, 117-125.

Fox, G. D., \& Holland, P. C. (1998). Neurotoxic hippocampal lesions fail to impair reinstatement of an appetitively conditioned response. Behavioral Neuroscience, 112, 255-260.

Gallistel, C. R., \& GibBon, J. (2000). Time, rate, and conditioning. Psychological Review, 107, 289-344.

GiBbON, J., \& BALSAM, P. D. (1981). Spreading associations in time. In C. M. Locurto, H. S. Terrace, \& J. Gibbon (Eds.), Autoshaping and conditioning theory (pp. 219-253). New York: Academic Press.

Haselgrove, M., \& Pearce, J. M. (2003). Facilitation of extinction by an increase or decrease in trial duration. Journal of Experimental Psychology: Animal Behavior Processes, 29, 153-166.

HoLLAND, P. C. (2000). Trial and intertrial durations in appetitive conditioning in rats. Animal Learning \& Behavior, 28, 121-135.

Kamin, L. J. (1969). Predictability, surprise, attention, and conditioning. In R. Church \& B. Campbell (Eds.), Punishment and aversive behavior (pp. 279-296). New York: Appleton-Century-Crofts.

KaYe, H., \& Mackintosh, N. J. (1990). A change of context can enhance performance of an aversive but not of an appetitive conditioned response. Quarterly Journal of Experimental Psychology, 42B, 113134.

KirkPATRICK, K., \& ChURCh, R. M. (2000). Independent effects of stimulus and cycle duration in conditioning: The role of timing processes. Animal Learning \& Behavior, 28, 373-388.

LatTal, M. K. (1999). Trial and intertrial durations in Pavlovian conditioning: Issues of learning and performance. Journal of Experimental Psychology: Animal Behavior Processes, 25, 433-450.

PaVLov, I. P. (1927). Conditioned reflexes (G. V. Anrep, Trans). London: Oxford University Press.

Pearce, J. M., \& Hall, G. (1980). A model for Pavlovian learning: Variations in the effectiveness of conditioned but not of unconditioned stimuli. Psychological Review, 87, 532-552.

Pfautz, P. L., \& Wagner, A. R. (1976). Transient variations in responding to Pavlovian conditioned stimuli have implications for the mechanisms of priming. Animal Learning \& Behavior, 4, 107-112.

RANDICH, A., \& Ross, R. T. (1985). Mechanisms of blocking by contextual stimuli. Learning \& Motivation, 15, 106-117.

Rescorla, R. A., \& Durlach, P. J. (1987). The role of context in intertrial interval effects in autoshaping. Quarterly Journal of Experimental Psychology, 39B, 35-40.

Rescorla, R. A., \& Wagner, A. R. (1972). A theory of Pavlovian conditioning: Variations in the effectiveness of reinforcement and nonreinforcement. In A. H. Black \& W. F. Prokasy (Eds.), Classical conditioning II: Current research and theory (pp. 64-99). New York: Appleton-Century-Crofts.

Terrace, H. S., Gibbon, J., Farrell, L., \& Baldock, M. D. (1975). Temporal factors influencing the acquisition and maintenance of an autoshaped keypeck. Animal Learning \& Behavior, 3, 53-62.

TERRY, W. S. (1976). Effects of priming unconditioned stimulus representation in short-term memory on Pavlovian conditioning. Journal of Experimental Psychology: Animal Behavior Processes, 2, 354269

WAGNER, A. R. (1978). Expectancies and the priming of STM. In S. H. Hulse, H. Fowler, \& W. K. Honig (Eds.), Cognitive processes in animal behavior (pp. 177-209). Hillsdale, NJ: Erlbaum.

WAGNER, A. R. (1981). SOP: A model of automatic memory processing in animal behavior. In N. E. Spear \& R. R. Miller (Eds.), Information processing in animals: Memory mechanisms (pp. 5-47). Hillsdale, NJ: Erlbaum.

WhitLOW, J. W., JR. (1975). Short-term memory in habituation and dishabituation. Journal of Experimental Psychology: Animal Behavior Processes, 1, 189-206.

(Manuscript received August 13, 2003; revision accepted for publication October 27, 2003.) 